LETTER TO THE EDITOR

\title{
Anisotropic universes with isotropic cosmic microwave background radiation
}

\author{
W C Lim, U S Nilsson and J Wainwright \\ Department of Applied Mathematics \\ University of Waterloo \\ Waterloo, Ontario \\ Canada N2L 3G1
}

\begin{abstract}
We show the existence of spatially homogeneous but anisotropic cosmological models whose cosmic microwave background temperature is exactly isotropic at one instant of time but whose rate of expansion is highly anisotropic. The existence of these models shows that the observation of a highly isotropic cosmic microwave background temperature cannot alone be used to infer that the universe is close to a Friedmann-Lemaitre model.
\end{abstract}

PACS numbers: 04.20.-q, 98.80.Dr

Submitted to: Class. Quantum Grav. 
It is widely believed by cosmologists that the universe can be accurately described by a Friedmann-Lemaitre (FL) model on sufficiently large scales. This belief stems from the fact that the temperature of the cosmic microwave background (CMB) is measured to be highly isotropic over the celestial sphere. In addition, certain theoretical results lend credence to this notion. The fundamental theorem due to Ehlers, Geren \& Sachs (1968), the so-called EGS theorem, states that if all fundamental observers measure an exactly isotropic CMB temperature during some time interval in an expanding universe, then the universe is exactly an FL model during this time interval. This theorem has been generalized by Stoeger et al (1995), and subsequently by Maartens et al (1995a, 1995b), to the "almost EGS theorem", which states that if all fundamental observers measure the CMB temperature to be almost isotropic during some time interval in an expanding universe, then the universe is described by an almost FL model during this time interval. In particular, the theorem implies that the overall expansion of the universe must be highly isotropic in the following sense. The anisotropy in the expansion is described by the shear scalar $\Sigma$, defined by

$$
\Sigma^{2}=\frac{\sigma_{a b} \sigma^{a b}}{6 H^{2}},
$$

where $\sigma_{a b}$ is the rate of shear tensor and $H$ is the Hubble scalar (see Wainwright \& Ellis, p 18-19). The statement that the expansion is highly isotropic means that $\Sigma \ll 1$.

For our purposes, the detailed form of the primary assumption of each theorem is of importance. The EGS theorem requires the following hypothesis:

$\mathbf{I}_{1}$ (the isotropy condition):

All fundamental observers measure the CMB temperature to be exactly isotropic during a time interval $I$.

The almost EGS theorem requires the analogous hypothesis with "exactly" replaced by "almost":

$\mathbf{A} \mathbf{I}_{1}$ (the almost-isotropy condition):

All fundamental observers measure the CMB temperature to be almost isotropic during a time interval $I$.

In the application of these theorems, the time interval $I$ is usually identified with $t_{\mathrm{e}} \leq t \leq t_{\mathrm{o}}$, where $t_{\mathrm{e}}$ is the time of last scattering and $t_{\mathrm{o}}$ is the time of observation (see, for example, Stoeger et al (1995), p 1-2). Both theorems make use of kinetic theory for describing the photons of the CMB (see, for example, Sachs \& Ehlers (1971) for an introduction to relativistic kinetic theory), and the proofs follow from the EinsteinLiouville equations. The distribution function for the photon fluid is assumed to be either exactly isotropic (the EGS theorem) or almost isotropic (the almost EGS theorem). The multipoles of the CMB temperature anisotropy can subsequently be written in terms of the anisotropies of the distribution function (see equation (40) and (41) in Maartens et al (1995a)). It is also important to note that a number of technical assumptions about the behaviour of the derivatives of the multipoles of the CMB temperature anisotropy must be made in order to prove the almost EGS theorem (see, for example, equation (1), (2), (3), and (4) in Maartens et al (1995b)).

Although the EGS theorem and the almost EGS theorem shed considerable light on the relation between idealized $\mathrm{CMB}$ temperature observations and the geometry 
of cosmological models, they cannot be used to conclude that the physical universe is close to an FL model. The reason for this limitation is basically that the hypotheses $\mathbf{I}_{1}$ and $\mathbf{A} \mathbf{I}_{1}$ above include the stipulation "during a time interval $I$ ", whereas we can only observe the CMB temperature at one instant of time on a cosmological scale. This observational limitation also implies that the technical assumptions needed for the almost EGS theorem are not observationally testable. In view of this situation, it is of interest to ask what restrictions, if any, can be placed on the anisotropy in the rate of expansion, using the following much weaker hypotheses:

$\mathbf{I}_{2}$ (the weak isotropy condition):

All fundamental observers measure the CMB temperature to be isotropic at some instant of time $t_{\mathrm{o}}$.

$\mathbf{A I}_{2}$ (the weak almost-isotropy condition):

All fundamental observers measure the CMB temperature to be almost isotropic at some instant of time $t_{\mathrm{o}}$.

On the basis of continuity, we can argue that either of assumptions $\mathbf{I}_{2}$ and $\mathbf{A} \mathbf{I}_{2}$ imply that all fundamental observers will measure the CMB temperature to be almost isotropic in some time interval $I_{\delta}$ of length $\delta$ centered on $t_{\mathrm{o}}$. This time interval could, however, be much shorter than the time interval $I$ referred to in $\mathbf{I}_{1}$ and $\mathbf{A} \mathbf{I}_{1}$. Nevertheless, if the technical assumptions mentioned above concerning the almost EGS theorem were satisfied, this theorem would imply that during the time interval $I_{\delta}$ the model is close to an FL model, and hence that the rate of expansion is highly isotropic (i.e. that $\Sigma \ll 1$ ). Thus, it is reasonable to ask whether $\mathbf{I}_{2}$ and $\mathbf{A} \mathbf{I}_{2}$ imply that the overall expansion of the universe must be almost isotropic at time $t_{\mathrm{o}}$, without imposing any of the technical assumptions about the multipoles.

In this Letter we answer this question in the negative. In particular we show that for a given time $t_{\mathrm{o}}$, there are spatially homogeneous cosmological models such that at $t_{\mathrm{o}}$ the CMB temperature is measured to be isotropic by all fundamental observers, even though the overall expansion of the universe is highly anisotropic at $t_{\mathrm{o}}$.

We consider the class of non-tilted spatially homogeneous models whose threeparameter isometry group is of Bianchi type VIII, and which are also locally rotationally symmetric (LRS). Since we are modeling the universe since the time of last scattering, it is reasonable to consider models with dust as the source of the gravitational field. In order to calculate the temperature of the CMB in these models, we adopt the approach of Nilsson et al (1999) the orthonormal frame formalism and makes use of expansion-normalized variables (see Wainwright \& Ellis (1997), p 112, for a motivation of this normalization). We will use the combined gravitational and geodesic evolution equations for models of Bianchi class A (which includes Bianchi VIII), which are given by equations (24)-(30) in NUW. This approach, which is purely geometric, differs from the kinetic approach used in the EGS and almost EGS theorems in that effects of the CMB photons on the gravitational field are neglected. These effects, however, will not change the results in any significant way.

The temperature of the CMB in a non-tilted spatially homogeneous cosmological

$\ddagger$ From now on we will refer to this reference as NUW. 
model as function of angular position on the celestial sphere is given by 3

$$
T(\theta, \varphi)=T_{\mathrm{e}} \exp \left[-\int_{\tau_{\mathrm{e}}}^{\tau_{\mathrm{o}}}(1+s) d \tau\right]
$$

where

$$
s=\Sigma_{\alpha \beta} K^{\alpha} K^{\beta},
$$

(see equation (58) in NUW). Here $T_{\mathrm{e}}$ is the temperature of the CMB at the surface of last scattering, which is assumed to be the same for all photons of the CMB. The $K^{\alpha}$ are the direction cosines of a particular null geodesic, and are related to the usual spherical angles $\theta, \varphi$ according to

$$
K^{\alpha}\left(\tau_{\mathrm{o}}\right)=(\sin \theta \cos \varphi, \sin \theta \sin \varphi, \cos \theta) .
$$

The quantity $\Sigma_{\alpha \beta}=\sigma_{a b} / H$ is the expansion-normalized shear tensor, given by

$$
\Sigma_{\alpha \beta}=\operatorname{diag}\left(\Sigma_{+}+\sqrt{3} \Sigma_{-}, \Sigma_{+}-\sqrt{3} \Sigma_{-},-2 \Sigma_{+}\right) .
$$

The time variable $\tau$ is the Hubble time, which is related to the cosmological clock time $t$ according to

$$
\frac{d t}{d \tau}=H^{-1},
$$

where $H$ is the Hubble variable.

For LRS models, the shear is restricted by $\Sigma_{-}=0$, which, in conjunction with (5), implies that (3) simplifies to

$$
s=\left(1-3 K_{3}^{2}\right) \Sigma_{+} .
$$

The geodesic equation for $K_{3}$ for LRS models simplifies to

$$
K_{3}^{\prime}=3\left(1-K_{3}^{2}\right) K_{3} \Sigma_{+},
$$

(see equation $(24 \mathrm{~h})$ in NUW). The prime denotes differentiation with respect to $\tau$. Since neither (7) nor (8) involve the geodesic variables $K_{1}$ or $K_{2}$, we need not consider the evolution equations for these variables. It follows from (8) that if $K_{3}=0, \pm 1$ initially, it has these values throughout the entire evolution. We temporarily exclude these values.

To solve the differential equation (8), we introduce the new variable

$$
y=\frac{1}{K_{3}^{2}}-1,
$$

which transforms (8) into

$$
y^{\prime}=-6 \Sigma_{+} y .
$$

It then follows that

$$
y(\tau)=y_{\mathrm{e}} \mathrm{e}^{-6 \beta(\tau)},
$$

where

$$
\beta(\tau)=\int_{\tau_{\mathrm{e}}}^{\tau} \Sigma_{+} d \tau
$$

$\S$ This formula is only true for non-tilted spatially homogeneous models. It can, however, easily be generalized to tilted models. If the assumption of spatial homogeneity is dropped, the problem of finding the temperature of the CMB is significantly more complicated (see, for example, Dunsby (1997) for a covariant and gauge-independent approach). 
which we refer to as the integrated shear. Combining (9) and (10) with (7) leads to

$$
s=\frac{1}{6}\left(\frac{2}{y}-\frac{3}{1+y}\right) y^{\prime} .
$$

Integrating from $\tau_{\mathrm{e}}$ to $\tau_{\mathrm{o}}$ yields

$$
\int_{\tau_{\mathrm{e}}}^{\tau_{\mathrm{o}}} s d \tau=-\ln \left[\left(\frac{y_{\mathrm{o}}+1}{y_{\mathrm{e}}+1}\right)^{1 / 2}\left(\frac{y_{\mathrm{e}}}{y_{\mathrm{o}}}\right)^{1 / 3}\right] .
$$

We note that $y_{\mathrm{o}}=\tan ^{2} \theta$, which follows from (4) and (9), and that $y_{\mathrm{e}}$ can be expressed in terms of $y_{\mathrm{o}}$ using (11) with $t=t_{\mathrm{o}}$. On substituting these results in (2) we obtain

$$
T(\theta, \varphi)=T_{\mathrm{e}} \mathrm{e}^{-\Delta \tau} \frac{\mathrm{e}^{2 \beta_{\mathrm{o}}}}{\sqrt{\mathrm{e}^{6 \beta_{\mathrm{o}}}+\left(1-\mathrm{e}^{6 \beta_{\mathrm{o}}}\right) \cos ^{2} \theta}},
$$

where $\Delta \tau=\tau_{\mathrm{o}}-\tau_{\mathrm{e}}$ and $\beta_{\mathrm{o}}=\beta\left(\tau_{\mathrm{o}}\right)$. It is easily verified that (15) also holds for the exceptional values $K_{3}=1,0,-1$, which correspond to $\theta=0, \frac{\pi}{2}$, $\pi$. Equation (15) shows explicitly that in the present class of cosmological models, the angular dependence of the CMB temperature is determined by the integrated shear. We emphasize that (15) holds independently of the field equations.

We now make use of the evolution equation for $\Sigma_{+}$(equation (24a) in NUW). With the LRS restrictions $\Sigma_{-}=0$ and $N_{1}=N_{2}$, this equation simplifies to

$$
\Sigma_{+}^{\prime}=-(2-q) \Sigma_{+}-\mathcal{S}_{+},
$$

where $q$ is the deceleration parameter, given by

$$
q=\frac{1}{2}\left(1-\frac{1}{12}\left(N_{3}^{2}-4 N_{2} N_{3}\right)\right)+\frac{3}{2} \Sigma_{+}^{2},
$$

and

$$
\mathcal{S}_{+}=-\frac{1}{3}\left(N_{3}^{2}-N_{2} N_{3}\right)
$$

is a component of the anisotropic spatial curvature. For Bianchi VIII LRS models, the spatial curvature variables satisfy $N_{2} N_{3}<0$, which implies that $\mathcal{S}_{+}<0$. In addition, since $\Sigma_{+}^{2} \leq 1$, it follows from (17) that $q \leq 2$. As a result of these inequalities, (16) implies that if $\Sigma_{+} \leq 0$ then $\Sigma_{+}^{\prime}>0$. Thus, any orbit that lies in the subset $\Sigma_{+}<0$ in state space will eventually cross the surface $\Sigma_{+}=0$, and enter the subset $\Sigma_{+}>0$. In other words, for an open set of orbits in the state space, $\Sigma_{+}$changes sign from negative to positive. It follows that for any such orbit we can choose $\tau_{0}$, keeping $\Delta \tau$ constant, so that the integrated shear $\beta\left(\tau_{\mathrm{o}}\right)$, as given by (12), is zero. It follows from (15) that

$$
T(\theta, \varphi)=T_{\mathrm{e}} \mathrm{e}^{-\Delta \tau}
$$

i.e., that the temperature at this time is completely isotropic. We summarize our results in the following theorem.

\section{Theorem 1}

There exist spatially homogeneous but anisotropic universes with the following properties:

(i) the CMB temperature is completely isotropic at some instant of time $t_{\mathrm{o}}$,

\| A detailed analysis of the orbits in fact shows that for almost all orbits, $\lim _{\tau \rightarrow-\infty} \Sigma_{+}=-1$ and $\lim _{\tau \rightarrow+\infty} \Sigma_{+}=\frac{1}{2}$. 
(ii) at time $t_{\mathrm{o}}$, the shear parameter $\Sigma_{0}$ satisfies $\Sigma_{0} \geq b$, where $b$ is a positive constant.

The theorem asserts that there are Bianchi VIII LRS models whose CMB temperature evolve in the following way. As the universe expands after last scattering, the $\mathrm{CMB}$ temperature reaches a state of maximum anisotropy, then becomes completely isotropic at time $t_{\mathrm{o}}$ and subsequently becomes increasingly anisotropic again. On the other hand the shear scalar $\Sigma$ becomes zero at some instant of time prior to $t_{\mathrm{o}}$, and then increases again, exceeding some value $b$ at time $t_{\mathrm{o}}$. A detailed analysis of the state space shows that the value of the constant $b$ depends on the value of the density parameter $\Omega_{\mathrm{o}}$ at the time of observation, and is not small unless $\Omega_{\mathrm{o}} \approx 1$, in which case the model is close to the flat FL model. The time $t_{\mathrm{o}}$, which we regard as the present age of the universe, is freely specifiable, since a point in state space determines the physical time only up to a multiplicative constant (see Wainwright \& Ellis, p 117).

We emphasize that this theorem does not contradict the almost EGS theorem, since the technical assumptions on the multipoles are not satisfied, as we now show. If $\tau$ is sufficiently close to $\tau_{\mathrm{o}}$ so that $\beta(\tau) \ll 1$, the quadrupole component of the temperature anisotropy can be written as $a_{2}=\left|a_{20}\right|$, where

$$
a_{20}=4 \sqrt{\frac{\pi}{5}}\left[\beta-\frac{1}{7} \beta^{2}+\mathcal{O}\left(\beta^{3}\right)\right] .
$$

This result follows from (15) with $\beta_{\mathrm{o}}$ replaced by $\beta(\tau)$, using equation (62) in NUW. By differentiating (20) with respect to $\tau$, and using the result $\beta^{\prime}(\tau)=\Sigma_{+}$, which follows from (12), we obtain the following evolution equation for the quadrupole moment $a_{2}$

$$
a_{20}^{\prime}=4 \sqrt{\frac{\pi}{5}}\left[1-\frac{2}{7} \beta+\mathcal{O}\left(\beta^{2}\right)\right] \Sigma_{+} .
$$

The above equation is, to lowest order, equivalent to the evolution equation for the quadrupole that arises in the kinetc theory based approach of Maartens et al (1995a) (differentiate equation (41) in that paper with respect to $\tau$ and use equations (12) and (14) in the same paper). From (21) we see that when $\beta=0=\beta_{\mathrm{o}}$, i.e. when the temperature of the CMB is perfectly isotropic over the celestial sphere, the derivative of $a_{2}$ is proportional to $\Sigma_{+}$. Therefore, since $\Sigma_{+}$, in general, will not be small, the derivative of $a_{2}$ will not be small either. Hence the technical assumptions of the almost EGS theorem are not satisfied.

It is also worth noting that the proof of the theorem does not depend on the full set of field equations (equations (24a)-(24d) in NUW, with $\Sigma_{-}=0$ and $N_{1}=N_{2}$ ), but only on the fact that the evolution equation (10) for the shear contains a driving term that has constant sign over the state space, as does $-\mathcal{S}_{+}$. The theorem thus applies to spatially homogeneous LRS cosmologies with sources more complicated than dust. For example, in Bianchi I LRS models with a homogeneous and source-free magnetic field, the evolution equation for $\Sigma_{+}$is of the form

$$
\Sigma_{+}^{\prime}=-(2-q) \Sigma_{+}+2 \mathcal{H}^{2}
$$

where $\mathcal{H}$ is the expansion-normalized magnetic field. Since this is of the same form as (16) and the geodesic equation (8) is exactly the same, the temperature of the CMB for these models will be the same as in (15) (9. Thus there exist magnetic cosmologies

I Since the Bianchi I LRS models with a homogeneous magnetic field are closely related to the Bianchi II LRS models, which are an invariant subset of the Bianchi VIII LRS models studied in this paper (see Collins (1972)), this result is not surprising. 
of this type whose CMB temperature is exactly isotropic at some time, but whose magnetic field and shear is not small. In other words, an almost isotropic CMB temperature cannot be used to limit a cosmological magnetic field, unless additional assumptions about the nature of the multipoles are made. This possibility seems to have been overlooked in Barrow (1997).

\section{Acknowledgements}

The research was supported in part by a grant from the Natural Sciences \& Engineering Research Council of Canada (JW), Gålöstiftelsen (USN), Svenska Institutet (USN), Stiftelsen Blanceflor (USN) and the University of Waterloo (USN, WCL).

\section{References}

Barrow J D 1997 Cosmological limits on slightly skew stresses Phys. Rev. D 557451

Collins C B 1972 Qualitative magnetic cosmology Commun. Math. Phys. 2737

Dunsby P K S 1997 A fully covariant description of CMB anisotropies Class. Quantum Grav. 14 3391

Ehlers J, Geren P, and Sachs R K 1968 Isotropic solutions of the Einstein-Liouville equations $J$. Math. Phys. 91344

Maartens R, Ellis G F R, and Stoeger W R 1995a Limits on anisotropy and inhomogeneity from the cosmic background radiation Phys. Rev. D 511525

Maartens R, Ellis G F R, and Stoeger W R 1995b Improved limits on anisotropy and inhomogeneity from the cosmological background radiation Phys. Rev. D 515942

Nilsson U S, Uggla C, and Wainwright J (NUW) 1999 A dvnamical svstems approach to geodesics in Bianchi cosmologies, to appear in Gen. Rel. Grav. gr-qc/9908062)

Sachs R K and Ehlers J 1971 Kinetic theory and cosmology in "Astrophysics and General Relativity" 1968 Brandeis University Summer Institute in Theoretical Physics, Vol. 2, Chretien M, Deser S, and Goldstein J Eds. (Gordon \& Breach:London)

Stoeger W R, Maartens R, and Ellis G F R 1995 Proving almost-homogeneity of the universe: an almost Ehlers-Geren-Sachs theorem Astrophys. Journ. 4411

Wainwright J and Ellis G F R 1997 Dynamical systems in cosmology (Cambridge University Press: Cambridge) 